\title{
Dossier thématique : maladie de Verneuil
}

\section{Thematic File: Verneuil's Disease}

\author{
A. Senéjoux \\ (C) Lavoisier SAS 2016
}

La maladie de Verneuil (MV), du nom du chirurgien qui l'a décrite en 1854, ou hidrosadénite suppurée, est considérée comme une maladie orpheline, elle n'est pourtant pas rare ; c'est une cause fréquente de suppuration anopérinéale puisque cette affection représentait environ $5 \%$ des causes de suppuration anopérinéale dans la série historique de Léopold Bellan.

Cette affection peut considérablement altérer la qualité de vie des patients touchés. Le traitement de référence demeure chirurgical, les alternatives médicales étant limitées et surtout représentées par les antibiotiques.

La physiopathologie de la maladie est encore mal connue mais des progrès récents dans sa compréhension permettent d'expliquer le développement de nouvelles cibles thérapeutiques.

Cette affection suscite un regain d'intérêt et l'espoir des patients à l'occasion de la mise sur le marché d'un nouveau traitement. Un symposium lui a été consacré lors des Journées de novembre 2015 de la Société nationale française de coloproctologie (SNFCP). Ce dossier a été entièrement rédigé d'après les communications de ce symposium.

Les flash conférences de ce congrès peuvent être revues dans leur intégralité en ligne sur le site de la SNFCP (http:// www.snfcp.org/congres/les-congres-passes/congres-passes/ article.phtml?id=rc/org/snfcp/htm/Article/2016/20160125085455-943).

\footnotetext{
A. Senéjoux $(\square)$

6, boulevard de la Boutière, F-35760 Saint Grégoire

e-mail : agnes.senejoux@gmail.com
} 